\title{
Dust Agglomeration in an Electrostatic Precipitator
}

\section{Aerosol and Air Quality Research}

\section{OPEN ACCESS}

Received: June 15, 2021

Revised: July 8, 2021

Accepted: July 14, 2021

\section{${ }^{*}$ Corresponding Authors:}

Kangping Cui

cuikangping@163.com

Ya-Fen Wang

yfwang@cycu.edu.tw

\section{Publisher:}

Taiwan Association for Aerosol Research

ISSN: $1680-8584$ print

ISSN: 2071-1409 online

Copyright: The Author(s). This is an open access article distributed under the terms of the Creative Commons Attribution License (CC BY 4.0), which permits unrestricted use, distribution, and reproduction in any medium, provided the original author and source are cited.

\author{
Kaijie Xu${ }^{1}$, Kangping Cui ${ }^{1 *}$, Jin-Luh Mou' ${ }^{2}$, Ya-Fen Wang ${ }^{3,4^{*}}$, Lin-Chi Wang ${ }^{3,4}$ \\ ${ }^{1}$ School of Resources and Environmental Engineering, Hefei University of Technology, Hefei \\ 230009, China \\ ${ }^{2}$ Liaocheng Baijun Environmental Technology Co., Ltd., Liaocheng 252100, China \\ ${ }^{3}$ Department of Environmental Engineering, Chung Yuan Christian University, Taoyuan 32023, \\ Taiwan \\ ${ }^{4}$ Center for Environmental Risk Management, Chung Yuan Christian University, Taoyuan 32023, \\ Taiwan
}

\section{ABSTRACT}

Dust agglomeration in an electrostatic precipitator (EP) during the sintering process taking place in an ironmaking plant was investigated. The dust agglomeration in the high sulfur flue gas dust hopper of the EP was very serious and did affect the removal of the dust from the hopper. The size of the agglomerate ranged from approximately $30-50 \mathrm{~cm}$ and was found to exhibit strong magnetic behavior. After combining actual operational observations and the physical and chemical test results, it was determined that the main factor resulting in the dust agglomeration in the EP was the unburned carbon, which was between $0.025 \%$ and $4.45 \%$ in the four samples of EP dust taken from different locations in the EP. In order to prevent fire accidents and dust agglomeration in the EP during the sintering process, the percentage of fine fuel particles (sized smaller than $1 \mathrm{~mm}$ ) should be minimized; the average fuel particle size for the sintering process should be controlled at $2.5-3.0 \mathrm{~mm}$, where the mass fraction accounts for approximately $80 \%$ of the fuel. In addition, strict sealing of the air box flue, the import and export flange, the expansion joint connection, the manhole door, and EP body should be carried out and maintained. Finally, the thickness of the bedding material should be more than $30 \mathrm{~mm}$ to ensure full combustion of the fuel used in the sintering process. This study provides useful information for the prevention of dust agglomeration in the EP during the sintering process.

Keywords: Dust, Agglomeration, Electrostatic precipitator, Sintering, Air pollution

\section{INTRODUCTION}

In recent years, the rapid development of modern industry has greatly improved the living standard of human beings, but at the same time, the flue gas emissions caused by production have seriously polluted the atmospheric environment and threatened human health (Shin et al., 2019). Therefore, more stringent industrial flue gas emission standards have been promulgated, and the application of various air pollution control devices has also received continuous attention (Lighty et al., 2000; Kim et al., 2015). Electrostatic precipitation (EP) is an efficient dust removal process (Zheng et al., 2019), where flue gas passes through a high-voltage electrostatic field driven by negative pressure, and the dust-containing gas is ionized and causes the dust to be charged. Under the action of an electric field force, the dust with a negative charge moves to the positive electrode (dust collector). When the dust reaches the positive electrode plate, it releases charges and adheres on the electrode. Then, the dust on the electrode drops into the dust hopper by tapping, and the purified flue gas is discharged into the atmosphere through a chimney (Beavis, 1993; Adabara et al., 2017; Dong et al., 2018; Gao et al., 2020). EPs (electrostatic precipitators) have many advantages, including high dust removal efficiency, low resistance, low energy consumption, efficient collection of dust in a large amount of flow gas at high temperatures, high automation, and easy maintenance, and the fact that they can be applied in cases involving 
corrosive gas. Therefore, they are widely used in electric power, smelting, cement, and other industrial fields (Jedrusik and Swierczok, 2009; Jaworek et al., 2018).

Generally, at the beginning of EP operations, the actual dust removal efficiency of the equipment is higher than the design value. However, as operating time is extended, the dust removal efficiency of the equipment decreases, gradually, which is directly or indirectly caused by different reasons, such as the uneven distribution of airflow in the electric field, the physical deformation of the cathode and anode framework, the disconnection of the cathode line, and the blockages caused by poor ash discharge from the ash hopper, which will further cause regional air pollution. Among these issues, the problem of ash hopper clogging occurs from time to time in dust removal systems in various industrial fields, which greatly affected the stable EP operations and the emissions of flue gas. According to previous studies, the causes of ash hopper blockages are as follows:1) parts of the dust collector (such as baffles, hammers, etc.) fall off, resulting in dust hopper nozzle blockages, 2) poor vibration effects; 3 ) high moisture content or condensation inside the precipitator leads to difficulties in dust removal, and 4) there is dust caking on the inner wall of the ash hopper (Shi et al., 2006; Saleem and Krammer, 2007; Jaworek et al., 2018). Usually, blockages caused by equipment problems can be resolved by quickly replacing spare parts and will not cause serious ash plugging in the ash hopper. However, ash hopper clogging caused by factors such as temperature, humidity, and operating parameters need to be comprehensively evaluated, and targeted adjustment measures should be taken.

As an example in this study, an EP at the sintering machine head in an ironmaking plant was operating smoothly. After approximately 100 days of operation, when the workers overhauled the equipment, they found that there were agglomerates in the ash hopper accompanied by ash blockage. Combined with actual operational observations and the physical and chemical test results, in this study, a comprehensive analysis of the causes of dust hopper blockage was carried out, for which reasonable and effective solutions are proposed. The results of this study provide useful information for the prevention of accidental fires and dust agglomeration in EP operations as well as strategies for air pollution control.

\section{ELECTROSTATIC PRECIPITATOR}

\subsection{Main Operational Parameters of an Electrostatic Precipitator}

The flue gas from the sintering machine was sucked into two separated stream flue gases. One was high in sulfur (350-450 ppm), and one had a low sulfur concentration (50-60 ppm). In the present study, gas streams with a total flow rate of 650 thousand $\mathrm{Nm}^{3} \mathrm{~h}^{-1}$ were flowed into an $\mathrm{EP}$, a de-SO $\mathrm{SO}_{\mathrm{x}}$ a SCR de-NO $\mathrm{N} /$ de-DXNs unit, and emitted from a stack, separately. The EP had electrode plates 15 meters long, $480 \mathrm{~mm}$ wide, and $1.5 \mathrm{~mm}$ thick, and the distance between the two electrode plates was $450 \mathrm{~mm}$. In addition, the operational electric field was $70-80 \mathrm{kV}$ and 1.2-1.5 A. There is another bag filter system to collect particulates from stock house, screen station, transfer towers and sinter plant building.

\subsection{Dust Agglomeration}

The EP operated well for approximately 100 days. On the $101^{\text {st }}$ day, during the maintenance of the system, the dust agglomeration in the high sulfur flue gas dust hopper of EP was very serious, and it did affect the dust removal operation from the hopper. The size of the agglomerate was approximately $30-50 \mathrm{~cm}$ (Fig. 1).

The agglomerate after cleaning with water is shown in Fig. 2. When the agglomerate was examined under a magnifying (20 times), the sintering phenomenon was observed. In addition, when the agglomerate was attracted by a magnet, it was found to exhibit strong magnetic behavior.

In order to determine the cause of the dust agglomeration in the EP, four samples $(X 1, X 2, X 3$, and $\mathrm{X} 4$ ) were taken and analyzed.

$\mathrm{X} 1$ : dust agglomerate from the hopper with the high sulfur gas stream

$\mathrm{X} 2$ : dust from the EP plates with the high sulfur gas stream

$X 3$ : normal powder dust from the hopper with the high sulfur gas stream

X4: normal powder dust from the hopper with the low sulfur gas stream. 


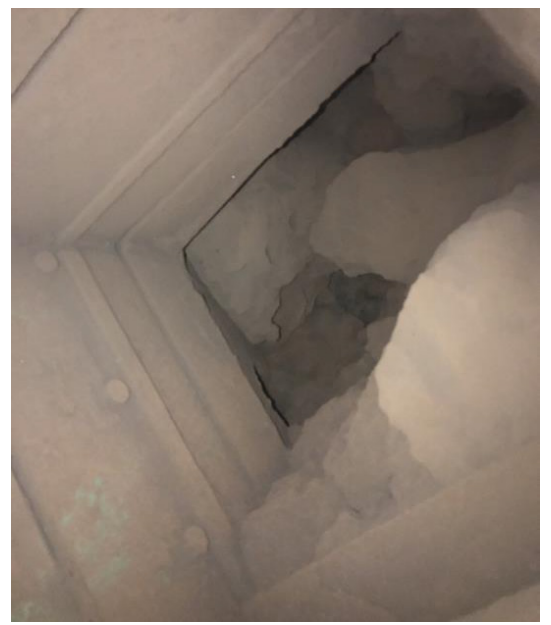

Fig. 1. The dust agglomerate in the ash hopper of an EP.

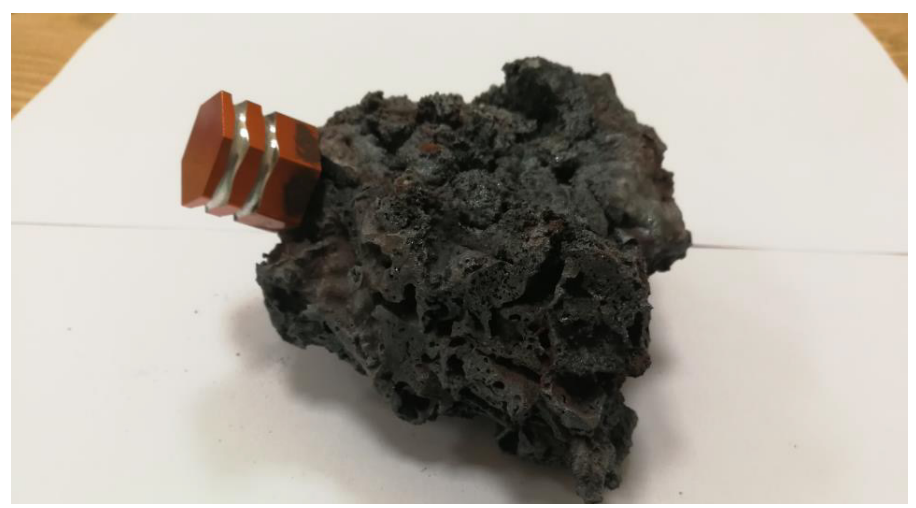

Fig. 2. The dust agglomerate after cleaning with water.

\section{RESULTS AND DISCUSSION}

The chemical composition of the samples is shown in Table 1.

Table 1 shows that the total iron (TFe) content in the four samples is all lower than that in the sintering ore. The carbon content of the sample is an important indicator. It can be seen that, among the 4 samples, the total carbon content (TC) in X2, X3, X4 is higher, and that of X1 (agglomerate) is significantly lower. The above results indicate that unburned carbon is ubiquitous in dust, and the carbon in the agglomerates was consumed during combustion. This also shows that the temperature level and the oxygen content in the ash hopper were both high enough to make the unburned carbon burn and further lead to agglomeration.

It can be seen from the profile diagram of the sintering bed (Fig. 3) that the high sulfur flue gas is located just inside the dry zone and the sparkling zone in the sintering bed, which is characterized by a high moisture content, a high dust concentration, a high exhaust gas temperature, and a high sulfur dioxide concentration. The dust contains unburned carbon, sinter debris, unreacted iron ore, and cosolvents (Shi et al., 2006). At this point, if enough oxygen is provided, it will lead to the combustion of any unburned carbon. The oxygen may come from the ash relief valve of the ash hopper, which allows air enter the ash hopper due to poor sealing. Sintered debris with sparks are carried into the ash hopper, which provides the temperature conditions necessary for smoldering. Under conditions with a high enough temperature and enough oxygen, unburned carbon begins to smolder in the ash hopper, resulting in serious agglomeration.

In order to further explore the crystal structure of the samples, we carried out an XRD analysis, as shown in Fig. 4. It can be seen from the XRD spectrum that no goethite (containing crystal water) diffraction peak was found in the agglomeration (X1), which indicates that the temperature of the 
Table 1. Results of the chemical composition analysis.

\begin{tabular}{llllll}
\hline & Sintering ore & X1 & X2 & X3 & X4 \\
\hline $\mathrm{TFe}$ & 58.5 & 55.56 & 51.53 & 52.60 & 47.21 \\
$\mathrm{CaO}$ & 9.7 & 5.88 & 5.44 & 5.65 & 7.99 \\
$\mathrm{MgO}$ & 1.06 & 1.22 & 0.82 & 0.97 & 1.14 \\
$\mathrm{Al}_{2} \mathrm{O}_{3}$ & 1.54 & 1.93 & 1.92 & 1.85 & 2.18 \\
$\mathrm{SiO}_{2}$ & 4.96 & 6.64 & 5.02 & 6.57 & 5.62 \\
$\mathrm{P}$ & 0.075 & 0.076 & 0.072 & 0.073 & 0.082 \\
$\mathrm{Oil}$ & - & $<0.01$ & 0.023 & 0.011 & - \\
$\mathrm{K} 2 \mathrm{O}$ & 0.027 & 0.12 & 0.15 & 0.41 & 0.85 \\
$\mathrm{Na} 2 \mathrm{O}$ & 0.022 & 0.041 & 0.037 & 0.054 & 0.038 \\
$\mathrm{T.C}$ & - & 0.025 & 3.34 & 1.92 & 4.45 \\
$\mathrm{~T} . \mathrm{S}$ & 0.005 & 0.31 & 1.38 & 0.67 & 0.60 \\
\hline
\end{tabular}

Note: Unit is \%.

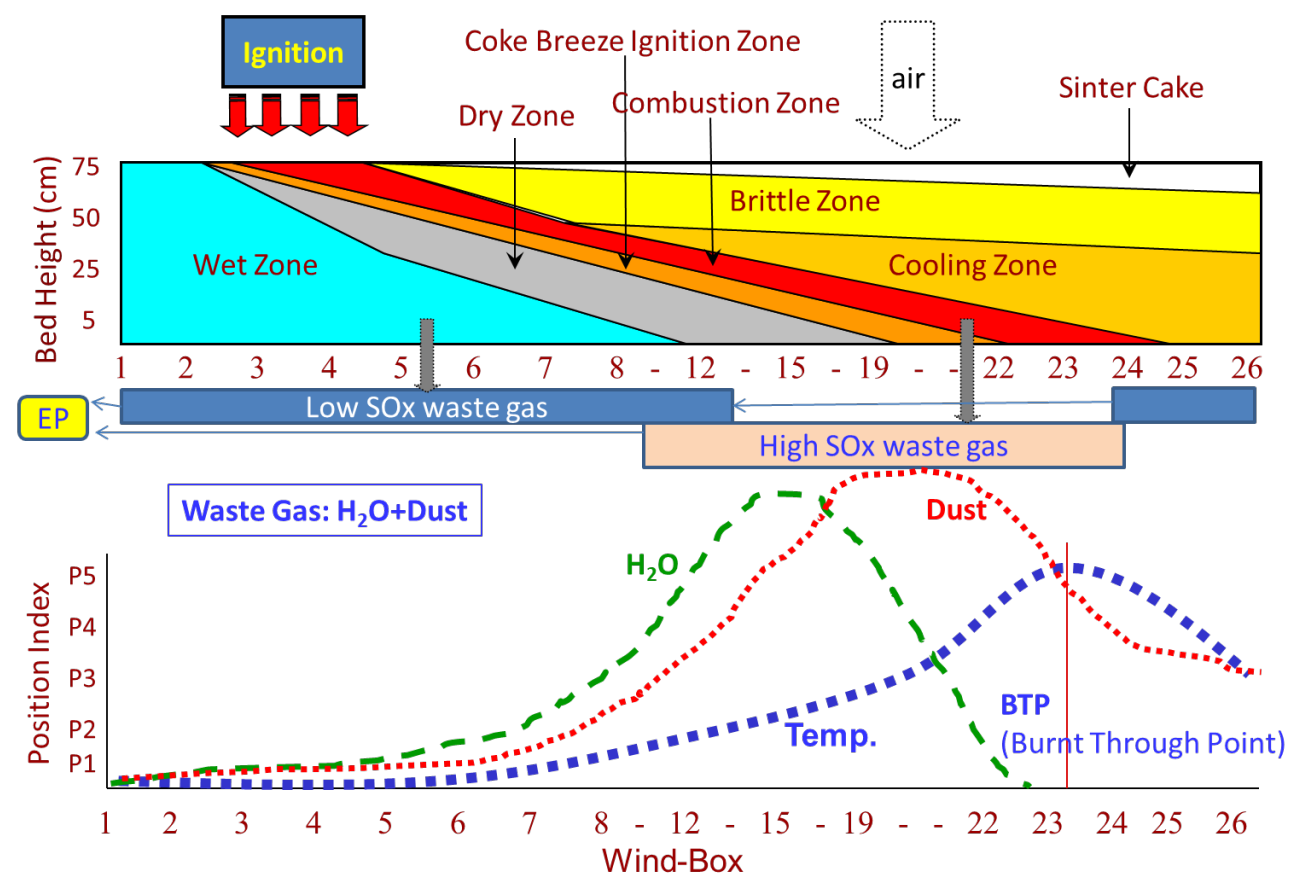

Fig. 3. Profile diagram of the sintering bed.

exhaust gas remained above the dew point $\left(130^{\circ} \mathrm{C}\right)$, so water is not the reason for the occurrence of agglomeration. At the same time, no sulfate was found in the agglomeration, indicating that $\mathrm{SO}_{\mathrm{x}}$ was not the main contributor to the agglomeration. In addition, the proportion of alkali metals $\left(\mathrm{Na}_{2} \mathrm{O}+\mathrm{K}_{2} \mathrm{O}\right)$ was also low, which indicated that this is also not the major cause of the agglomeration.

In the XRD pattern of the agglomerate, we found that the proportion of magnetite $\left(\mathrm{Fe}_{3} \mathrm{O}_{4}\right)$ was high, and the carbon content was low. This is because under a sufficient temperature and adequate oxygen, unburned carbon starts to burn in the ash hopper, releasing heat and generating CO. At a high temperature, hematite is reduced by $\mathrm{CO}$ to magnetite, and at the same time, the burning of the unburned carbon causes dust sintering in the ash hopper, which in turn results in dust agglomeration (Barranco et al., 2007; Kang et al., 2015).

In addition, by analyzing the particle size of the coke breeze during the operation, it was found that the proportion of coke breeze with particles sizes less than $1 \mathrm{~mm}$ was as high as $55 \%$, and the proportion of coke powder with particle sizes ranging from 1-3 mm was approximately $20 \%$. Previous research has indicated that the optimal size distribution of coke breeze is between 1.0 and $3 \mathrm{~mm}$ for $45-55 \%$, but other research has led to different results (Zhao et al., 2015; Lanzerstorfer and Steiner, 2016). However, there is no specific definition of size distribution. It depends on the 

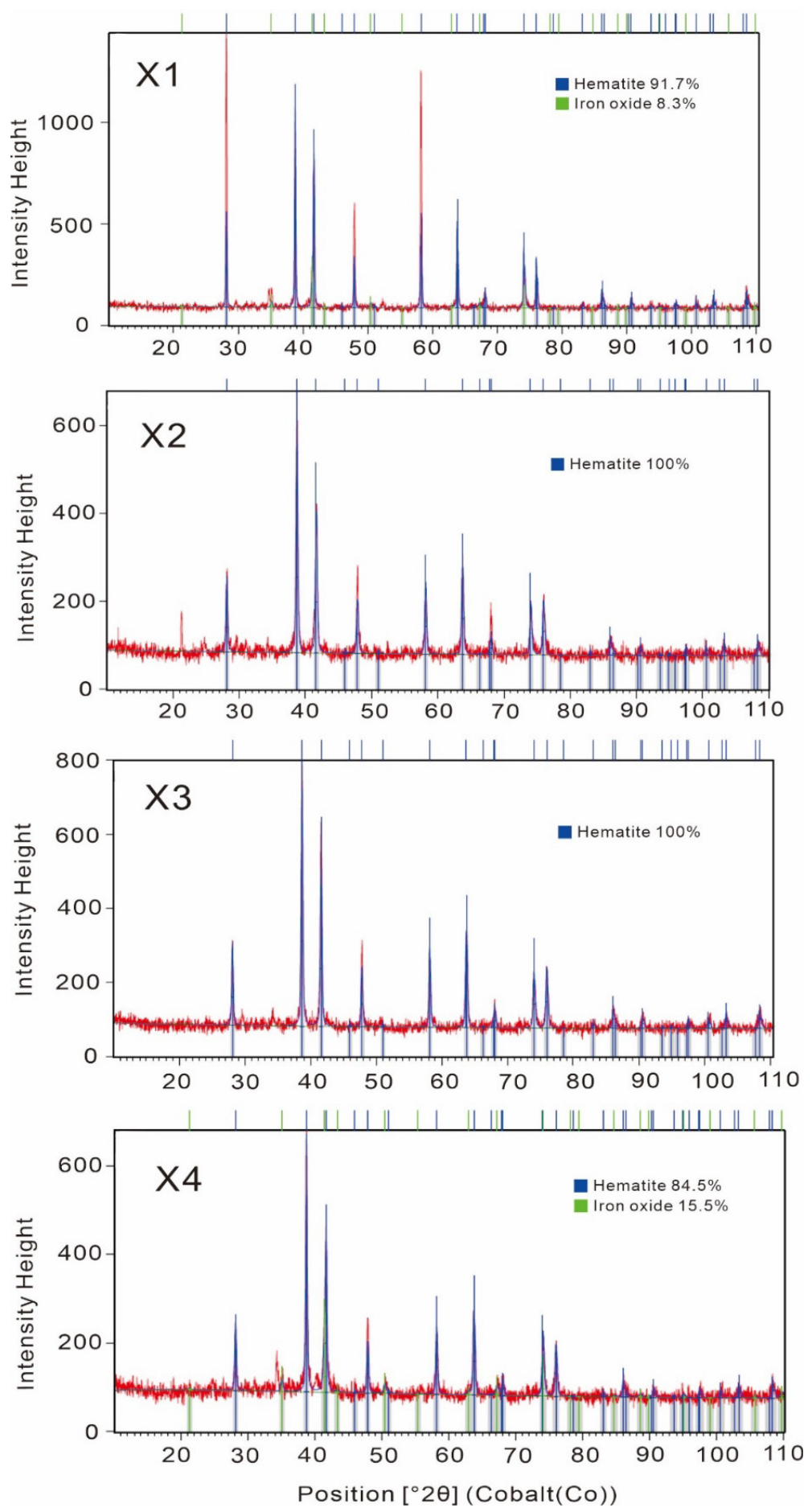

Fig. 4. XRD spectra of the samples.

sinter mix granulation effect. For example, when using a mini-pellet sintering process, a high ratio of limonite, or high iron concentrations, the moisture and size control are very different (Pahlevaninezhad et al., 2014; Zhao et al., 2015). Therefore, a granulation test is necessary to optimize the coke breeze size and determine the amount of water to be added.

To summarize, the formation of lumpy agglomerates inside the EP hopper can be summarized as follows:

1. The size distribution of the coke breeze did not go well with the water absorption properties of the sinter mix; therefore, the granulation did not make the coke fine attached onto the pseudo-particles. 
2. The un-attached coke fine was sucked into the wind-box, and more coke fine was used to compensate for the heat requirement in the process. Eventually, coarse coke segregated to the sinter bed bottom layer, and some flame sparks went through the wind-leg to the windbox and the into the EP.

3. With the high portion of $\mathrm{C}$ (coke fine) and high temperature sparks, smoldering occurred in the EP hopper under insufficient oxygen conditions. Finally, a lumpy magnetite agglomerate was generated.

\section{CONTROL MEASURES}

Combined with the results of physical and chemical analysis and evidence from practical operation, the following control measures are proposed:

\subsection{Strict Control of the Coke Particle Size}

For a traditional sinter plant, which uses Australian and Brazilian iron ore fine as the major raw materials, the optimal fuel size control is suggested to be less than $30 \%$ for $<1 \mathrm{~mm}$ portion, and around $45-55 \%$ for $1.0-3.0 \mathrm{~mm}$, and the mean size for $1.0-2.0 \mathrm{~mm}$.

Sinter plant was asked to use coke breeze together with Coke Water Quench (CWQ) sediment sludge, which composed of mainly fine particles, and hard to control the entire fuel size distribution. Therefore, to stop using CWQ sludge and continuous monitoring the fuel size is suggested.

\subsection{Strengthening the Management of Air Leakage Points}

Good sealing of the air box flue, the import and export flange, the expansion joint connection, the manhole door, and the EP body should be maintained. Fortunately, the lumpy magnetite agglomerate was found early. If a serious leakage leads to $\mathrm{O}_{2}$ in the hopper, then an EP fire could occur. After inspection, it was found that the plug valve of the ash hopper was seriously worn. It was suggested that the plug valve be replaced with a wear-resistant push welding plate. Furthermore, we strongly suggested analyzing the carbon content of EP dust, setting up C control limits, and periodic cleaning of the system.

\subsection{Adjusting the Thickness of the Hearth Layer}

The thickness of hearth layer should be adjusted to avoid sparks being brought into the dust collector. The thickness of the bedding material should be more than $40 \mathrm{~mm}$, and the particle size should be maintained at 10-20 mm (Zhao et al., 2015; Liu et al., 2016). The uniformity of the particle size and the thickness of the bedding material should be strictly controlled and adjusted on a timely basis to ensure complete combustion of the fuel and to protect the plug of grate bars.

\section{CONCLUSION}

In this study, dust agglomeration in an electrostatic precipitator (EP) during a sintering process in an ironmaking plant was investigated. The dust agglomeration in the high sulfur flue gas dust hopper of the EP was very serious, and it did affect the dust removal operation from the hopper. Combined with the actual operation observation and physical and chemical test results, this study made a comprehensive analysis of the reasons for dust agglomeration, and drew the following main conclusions.

1. The size of the dust agglomerate was approximately $30-50 \mathrm{~cm}$, and it was found to have strong magnetic behavior.

2. After combining actual operational observations with the physical and chemical test results, it was determining that the main factor resulting in the dust agglomeration in the EP was the unburned carbon content, which was between $0.025 \%$ and $4.45 \%$ in the four samples of the EP dust taken from various locations.

3. In order to prevent fire accidents and dust agglomeration in the EP during a sintering process, the percentage of fine fuel particle sizes smaller than $0.2 \mathrm{~mm}$ and less than $1 \mathrm{~mm}$ should be minimized. 
4. The average fuel particle size for a sintering process should be controlled at $1.0-2.0 \mathrm{~mm}$, and its mass fraction between $1-3 \mathrm{~mm}$ be account for approximately $45-55 \%$.

5. A good and strict sealing of the air box flue, the import and export flange, the expansion joint connection, the manhole door, and the EP body should be ensured and maintained.

6. The thickness of bedding material should not be less than $30 \mathrm{~mm}$ to ensure full combustion of fuel in the sintering process.

This study provided useful information for practical prevention of dust agglomeration in an EP during a sintering process.

\section{REFERENCES}

Adabara, I., Hassan, A.S. and Hassan, A.S. (2017). Design and implementation of an electrostatic precipitator and its cleaning system for small scale combustion. Indo-Iran. J. Sci. Res. 1, 213224.

Barranco, R., Gong, M., Thompson, A., Cloke, M., Hanson, S., Gibb, W., Lester, E. (2007). The impact of fly ash resistivity and carbon content on electrostatic precipitator performance. Fuel 86, 2521-2527. https://doi.org/10.1016/j.fuel.2007.02.022

Beavis, R.J. (1993). Dust Collectors. US

Dong, M., Zhou, F., Zhang, Y., Shang, Y., Li, S. (2018). Numerical study on fine-particle charging and transport behaviour in electrostatic precipitators. Powder Technol. 330, 210-218. https://doi.org/10.1016/j.powtec.2018.02.038

Gao, W., Wang, Y., Zhang, H., Guo, B., Zheng, C., Guo, J., Gao, X., Yu, A. (2020). A numerical investigation of the effect of dust layer on particle migration in an electrostatic precipitator. Aerosol Air Qual. Res. 20, 166-179. http://dx.doi.org/10.4209/aaqr.2019.11.0609

Jaworek, A., Sobczyk, A., Krupa, A., Marchewicz, A., Czech, T., Śliwiński, L. (2018). Hybrid electrostatic filtration systems for fly ash particles emission control. A review. Sep. Purif. Technol. 213, 283-302. https://doi.org/10.1016/j.seppur.2018.12.011

Jedrusik, M., Swierczok, A. (2009). The influence of fly ash physical and chemical properties on electrostatic precipitation process. J. Electrostat. 67, 105-109. https://doi.org/10.1016/j.elstat. 2008.12.014

Kang, L., Zhang, L., Zhang, D., Huang, J., Xue, G. (2015). Treatment and utility of the electrostatic precipitator dust of the sintering machine. industrial safety and environmental protection. 41, 41-43. https://doi.org/10.3969/j.issn.1001-425X.2015.03.013 (in Chinese)

Kim, K.H., Kabir, E., Kabir, S. (2015). A review on the human health impact of airborne particulate matter. Environ. Int. 74, 136-143. https://www.ncbi.nlm.nih.gov/pubmed/25454230

Lanzerstorfer, C., Steiner, D. (2016). Characterization of sintering dust collected in the various fields of the electrostatic precipitator. Environ. Technol. 37, 1559-1567. https://doi.org/10.108 0/09593330.2015.1120787

Lighty, J.S., Veranth, J.M., Sarofim, A.F. (2000). Combustion aerosols: Factors governing their size and composition and implications to human health. J. Air Waste Manage. Assoc. 50, 15651618. https://doi.org/10.1080/10473289.2000.10464197

Liu, C.X., Xie, Z.H., Sun, F.R., Chen, L.G. (2016). Optimization for sintering proportioning based on energy value. Appl. Therm. Eng. 103, 1087-1094. https://doi.org/10.1016/j.applthermaleng.2 016.04.158

Pahlevaninezhad, M., Emami, M.D., Panjepour, M. (2014). The effects of kinetic parameters on combustion characteristics in a sintering bed. Energy 73, 160-176. https://doi.org/10.1016/j.e nergy.2014.06.003

Saleem, M., Krammer, G. (2007). Effect of filtration velocity and dust concentration on cake formation and filter operation in a pilot scale jet pulsed bag filter. J. Hazard. Mater. 144, 677681. https://doi.org/10.1016/j.jhazmat.2007.01.094

Shi, Y., Da Ng, X.Q., Han, X.M., Wang, D. (2006). Characteristics \& applications of electrostatic precipitator for sintering dust. Heavy Mach. 2006, 27-30,34. (in Chinese)

Shin, D.H., Woo, C.G., Kim, H.J., Kim, Y.J., Han, B. (2019). Comparison of discharging electrodes for the electrostatic precipitator as an air filtration system in air handling units. Aerosol Air Qual. Res. 19, 671-676. http://dx.doi.org/10.4209/aaqr.2018.10.0367 
Zhao, J.P., Loo, C.E., Dukino, R.D. (2015). Modelling fuel combustion in iron ore sintering. Combust. Flame 162, 1019-1034. https://doi.org/10.1016/j.combustflame.2014.09.026

Zheng, C., Duan, D., Chang, Q., Liu, S., Yang, Z., Liu, X., Weng, W., Gao, X. (2019). Experiments on enhancing the particle charging performance of an electrostatic precipitator. Aerosol Air Qual. Res. 19, 1411-1420. https://doi.org/10.4209/aaqr.2018.11.0400 